reduction in frequency of incontinent episodes of 6 (8.25) to 2 (7.25), $p=0.029$. Two achieved complete continence. There was an increase in deferral time, 3 (4) to 5 (8) minutes, $p=0.027$.

Conclusion Daily bilateral transcutaneous PTNS is an effective treatment for FI. It can be easily self-administered from home.

Disclosure of Interest None Declared.

\section{PWE-015 PUDENDAL NERVE STIMULATION FOR FAECAL INCONTINENCE}

doi:10.1136/gutjnl-2013-304907.304

1." G Thomas, 'A George, 'T Dudding, 'R J Nicholls, ' $\mathrm{C}$ Vaizey. ${ }^{1}$ St Mark's Hospital and Academic Institute, London, UK

Introduction Sacral nerve stimulation (SNS) is an established treatment for faecal incontinence (FI). However, only $50-60 \%$ of patients will have a good response to SNS. Further treatment is difficult for those who do not respond to SNS. Pudendal nerve stimulation (PNS) was first reported for FI in 2005. Since then only one small study has further reported its use for FI. We aimed to assess PNS for FI in those who have failed to improve with SNS.

Methods Recruited patients underwent test PNS. Those who experienced $\mathrm{a} \geq 50 \%$ reduction in frequency of $\mathrm{FI}$ episodes underwent permanent PNS. The primary outcome measure was the change in frequency of FI episodes. Further outcome measures were further bowel diary data, St Marks FI Score, Rockwood FI QOL Score, SF-36 QOL Score and anorectal physiological changes.

Results Ten patients underwent test PNS. Five experienced a $\geq$ $50 \%$ improvement in frequency of FI episodes, and underwent permanent stimulation. One withdrew from the study at six months. At median follow up of 24 (3-36) months, the median frequency of FI improved from 5 (18.25) to 2.5 (3) per week; $p=0.043$. Three maintained $\mathrm{a} \geq 50 \%$ improvement in soiling. There was a significant improvement in the St Marks FI score, 19 (6) to 16 (4.5); $p=0.042$ There were no significant changes in the ability to defer defecation or in quality of life measures.

Conclusion PNS may be an effective treatment for FI for those who have failed to improve with SNS

Disclosure of Interest None Declared.

\section{PWE-016 CAN THE RESPONSE TO POSTERIOR TIBIAL NERVE STIMULATION FOR FAECAL INCONTINENCE PREDICT THE RESPONSE TO SACRAL NERVE STIMULATION?}

doi:10.1136/gutjnl-2013-304907.305

1."G Thomas, 'T Dudding, 'R J Nicholls, ' $\mathrm{C}$ Vaizey. ${ }^{1}$ St Mark's Hospital and Academic Institute, London, UK

Introduction Sacral nerve stimulation (SNS) is an established treatment for faecal incontinence (FI). Posterior tibial nerve stimulation (PTNS) is a more recent and increasingly popular treatment for FI. PTNS is cheaper, safer and as effective as SNS in the short term. PTNS can be used as an initial therapy, with SNS reserved for those who have failed to improve. The aim of this study was to see if the response to PTNS can be used to predict the response to SNS.

Methods Prospectively collected data for all patients who had undergone transcutaneous PTNS followed by SNS were analysed. A good clinical response to PTNS and to SNS was defined as a $\geq 50 \%$ reduction in frequency of FI episodes per week.

Results Ten patients underwent PTNS followed by SNS for FI. Three patients had a poor response to PTNS. Two of these had a subsequently had a poor response to SNS. Seven patients had a good response to PTNS. Five of these had a good response to SNS.
Conclusion This small study suggests that the response to PTNS may predict the response to SNS. Much larger studies are needed to explore this further

Disclosure of Interest None Declared.

\section{PWE-017 IS BOWEL SCREENING EFFECTIVE AT IDENTIFYING BOWEL CANCER EARLY?}

doi:10.1136/gutjnl-2013-304907.306

${ }^{1,}{ }^{*} \mathrm{H}$ Glover, ${ }^{2} \mathrm{~N}$ Townsend on behalf of Bowel Screening Wales, ${ }^{3} \mathrm{M}$ Guy on behalf of Cancer Services, ${ }^{4} \mathrm{D}$ Bowen. 'Gastroenterology; ${ }^{2}$ Bowel Screening UK, Glangwili Hospital; ${ }^{3}$ Cancer Services, Glangwili Hospial; ${ }^{4}$ Consultant Gastroenterologist, Glangwili Hospital, Carmarthen, UK

Introduction Bowel screening aims to identify bowel cancer early to achieve a lower mortality. We looked at whether patients diagnosed with cancer on a bowel screening list have disease at an earlier Dukes stage and thus better prognosis than those with symptoms.

Methods 176 patients with an endoscopic diagnosis of bowel cancer were identified retrospectively, 63 patients from the Bowel Screening Wales (BSW) list and 137 from all other lists. All patients underwent colonoscopy in Glangwili Hospital between Jan 2009 and Dec 2011. All cases had a histological diagnosis of bowel cancer and subsequent staging using the Dukes classification.

Results Results showed similar M:F ratios of 73:27\% (BSW cohort) and 63:37\% (symptomatic cohort). The median ages were 66 yrs (BSW) and 67 yrs (symptomatic). The age range for both groups was 60-72 yrs

The table below compares the no. of patients (\%) with each Dukes stage of disease and its statistical significance.

\section{Abstract PWE-017 Table}

\begin{tabular}{llll}
\hline Dukes Staging & No. of BSW pts (\%) & No. of symptomatic pts (\%) & P value \\
\hline A & $26(41 \%)$ & $33(24 \%)$ & 0.019 \\
B & $17(27 \%)$ & $51(37 \%)$ & 0.19 \\
C & $16(26 \%)$ & $25(18 \%)$ & 0.26 \\
D & $4(6 \%)$ & $28(21 \%)$ & 0.01 \\
Total pts & 63 & 137 & \\
\hline
\end{tabular}

A fishers analysis was carried out. Using a $\mathrm{P}$ value $<0.05$ there was a significant difference in the number of patients presenting at stages $A(p=0.019)$ and $D(p=0.01)$ and no statistical difference at stages $B(p=0.19)$ and $C(p=0.26)$.

In the BSW cohort 4 patients (6.4\%) had advance disease and were not fit for surgery compared to 12 patients (8.6\%) in the symptomatic cohort. The number of patients cured by polypectomy was $7(11.1 \%)$ in the BSW cohort and $1(0.7 \%)$ in the symptomatic group. 52 patients $(82.5 \%)$ had bowel resection in the BSW group and $124(90.5 \%)$ in the symptomatic group. The only group with a significant difference statistically was the number of patients treated with polypectomy $(p$ value $=0.0014)$. The number of patients in each group that were inoperable due to advanced disease or that went on to have bowel resection did not differ statistically with $p$ values of 0.78 and 0.16 respectively.

Data was also collected identifying the number of patients in each group by the site of bowel cancer. The distribution was similar in both groups.

Conclusion Our study demonstrates that more patients are identified with Dukes stage A $(p=0.019)$ disease through the bowel screening programme and that significantly more patients are managed by polypectomy alone $(p=0.0014)$. It also demonstrates that significantly less patients are diagnosed with Dukes stage $D(p=0.01)$ disease through bowel screening. This supports the role of bowel screening and its clinical role in reducing mortality from bowel cancer.

Disclosure of Interest None Declared. 